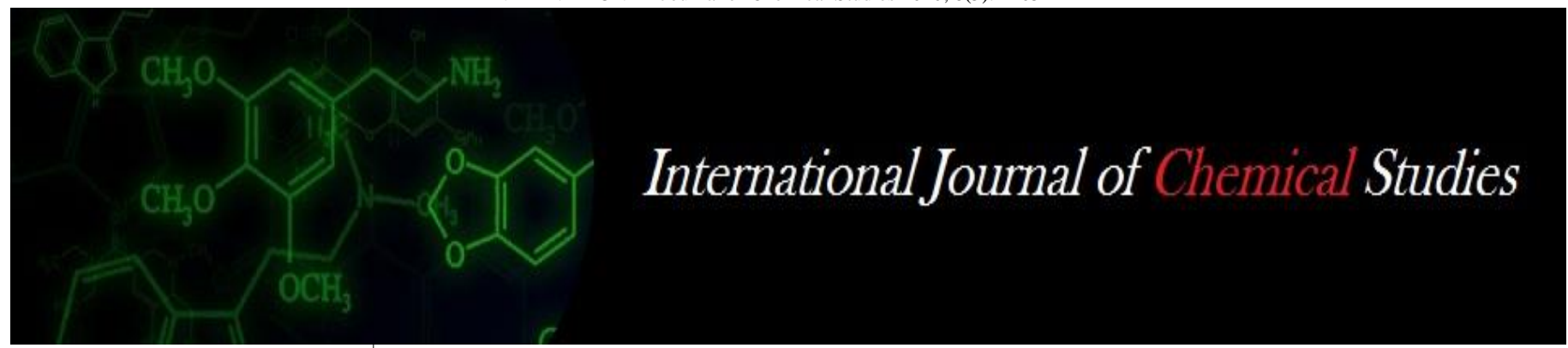

P-ISSN: 2349-8528

E-ISSN: 2321-4902

www.chemijournal.com

IJCS 2020; 8(5): 2105-2114

(C) 2020 IJCS

Received: 13-06-2020

Accepted: 18-07-2020

Asha Sinha

Department of Mycology \&

Plant Pathology, Institute of

Agricultural Sciences, B.H.U.,

Varanasi, Uttar Pradesh, India

Ravindra Kumar

a. Department of Mycology \&

Plant Pathology, Institute of

Agricultural Sciences, B.H.U.,

Varanasi, India

b. ICAR-Indian Institute of

Wheat and Barley Research,

Karnal, Haryana, India

Seweta Srivastava

School of Agriculture, Lovely

Professional University,

Phagwara, Punjab, India

Corresponding Author:

Seweta Srivastava

School of Agriculture, Lovely

Professional University,

Phagwara, Punjab, India

\section{Abundance, frequency and occurrence based comparison between mycoflora involved in the decomposition of Sesbania aculeata L. under experimental and natural conditions}

\author{
Asha Sinha, Ravindra Kumar and Seweta Srivastava
}

DOI: $\underline{\text { htps://doi.org/10.22271/chemi.2020.v8.i5ac.10613 }}$

\begin{abstract}
Green manuring is a cost-effective technology to sustain soil fertility and enhance activities of soil microflora and micro-fauna. The comparison between mycoflora associated with the decomposition of Sesbania aculeata L. green manure under nylon net bag and mycoflora inhabiting soil mixed green manure at experimental site was investigated. During the study abundance, frequency and occurrence of both type of mycoflora was investigated. Maximum moisture content was recorded in the month of July and thereafter it decreased. The average number of fungi involved in the decomposition of Sesbania aculeata $\mathrm{L}$. was found to be dependent on physical climate viz., moisture content, rainfall, temperature and relative humidity. The highest occurrence of mycoflora in experimental condition $\left(48.95 \times 10^{4}\right.$ per $\mathrm{g}$ green manure) and natural condition $\left(47.85 \times 10^{4}\right.$ per g green manure) was found in the month of July, whereas in the month of June lowest occurrence of mycoflora in both conditions was recorded. The number of fungi slightly decreased in August and thereafter declined gradually up to December. From the above finding, pattern of fungal succession on decomposing green manure was deciphered that may enable rapid decomposition of agricultural wastes.
\end{abstract}

Keywords: Decomposition, green manure, Mycoflora, Sesbania aculeata L. and soil

\section{Introduction}

The excessive application of synthetic agrochemicals has jeopardized the environment through soil, water and air contaminations and pollutions and has adversely affected the soil microflora by altering the chemical and physical properties of the soil. The pesticides residues in food chain have endangered the whole life sustaining system around the world (Singh et al., 2018) [30]. In recent years, however, greater regulation of agrochemicals and increasing costs of application are motivating the development of alternative crop management strategies. The use of green manuring may be a viable option to restrict the excessive application of fertilizers and other agrochemicals. Hence, there have been renewed research efforts during the past two decades on green manures (Sinha et al., 2009, Kumar et al., 2014a, b; Kumar et al., 2017; Kumar et al., 2020) $[31,20,19,17]$. However, crop intensification urgently needs a rapid decomposition of crop residues, green manures or organic bulks of agricultural wastes in the soil. As an alternative, burning of the crop residues is hazardous to soil health and polluting our environment drastically and thereby the decomposition of this material is one of the leading environmental concerns of today's world (Kumar et al., 2014a) ${ }^{[20]}$. Soil organisms carry a wide range of processors that are important for soil health in both natural and managed agricultural system. The population, diversity and activity of soil-biota will fluctuate as soil environment changes (Kumar et al., 2010) ${ }^{[18,24]}$. Therefore, the present study was undertaken to understand the changes in abundance, frequency and occurrence of fungal community involved in the decomposition of Sesbania aculeata L. green manure and their efficacy in the decomposition.

\section{Material and Methods}

A. Decomposition of Sesbania aculeata $\mathrm{L}$. under experimental conditions

Sesbania aculeata L. was sown to generate the research material at experimental farm of Institute of Agricultural Sciences, B.H.U. and at 45 DAS, the crop was cut down. 
50 nylon net bags (mesh size of $1 \mathrm{~mm}^{2}$ ) were prepared containing $50 \mathrm{~g}$ of air-dried green manure in each bag (size $30 \times 25 \mathrm{~cm}$ ) to facilitate the microbial decomposition and reduced the macro faunal disturbances.

These bags were buried in a trench with an area of $4 \times 4 \times 0.1 \mathrm{~m}$ in the field. Samples (4 bags) were drawn randomly from July, 2008 to June, 2009 at monthly intervals for isolation of mycoflora.

In natural condition, the green manure was allowed for decomposition after cutting down and mixing of the crop in the field.

The sampling programme was run monthly for litter analysis in both experimental and natural conditions.

Weight Loss: The weight loss of the green manure was determined by means of litter nylon net bag weight technique (Bocock and Gilbert, 1957) ${ }^{[4]}$.
In each month three bags were picked and fresh weight of the content was taken. The material was oven dried at $60^{\circ} \mathrm{C}$ for dry weight determination.

pH: The decomposing green manure mixed in distilled water in the ratio of 1:5 and thereafter the $\mathrm{pH}$ was determined with the help of Elico-Electric $\mathrm{pH}$ meter.

Moisture content: The samples of the decomposing green manure from the bags were oven dried at $60{ }^{\circ} \mathrm{C}$ for 24 hours and moisture content was determined on the basis of losses in dry weight.

Meteorological data: Meteorological data regarding maximum and minimum temperatures, relative humidity and rainfall were obtained from Department of Agronomy, Institute of Agricultural Sciences, Banaras Hindu University, Varanasi (UP), India (Fig. 1).

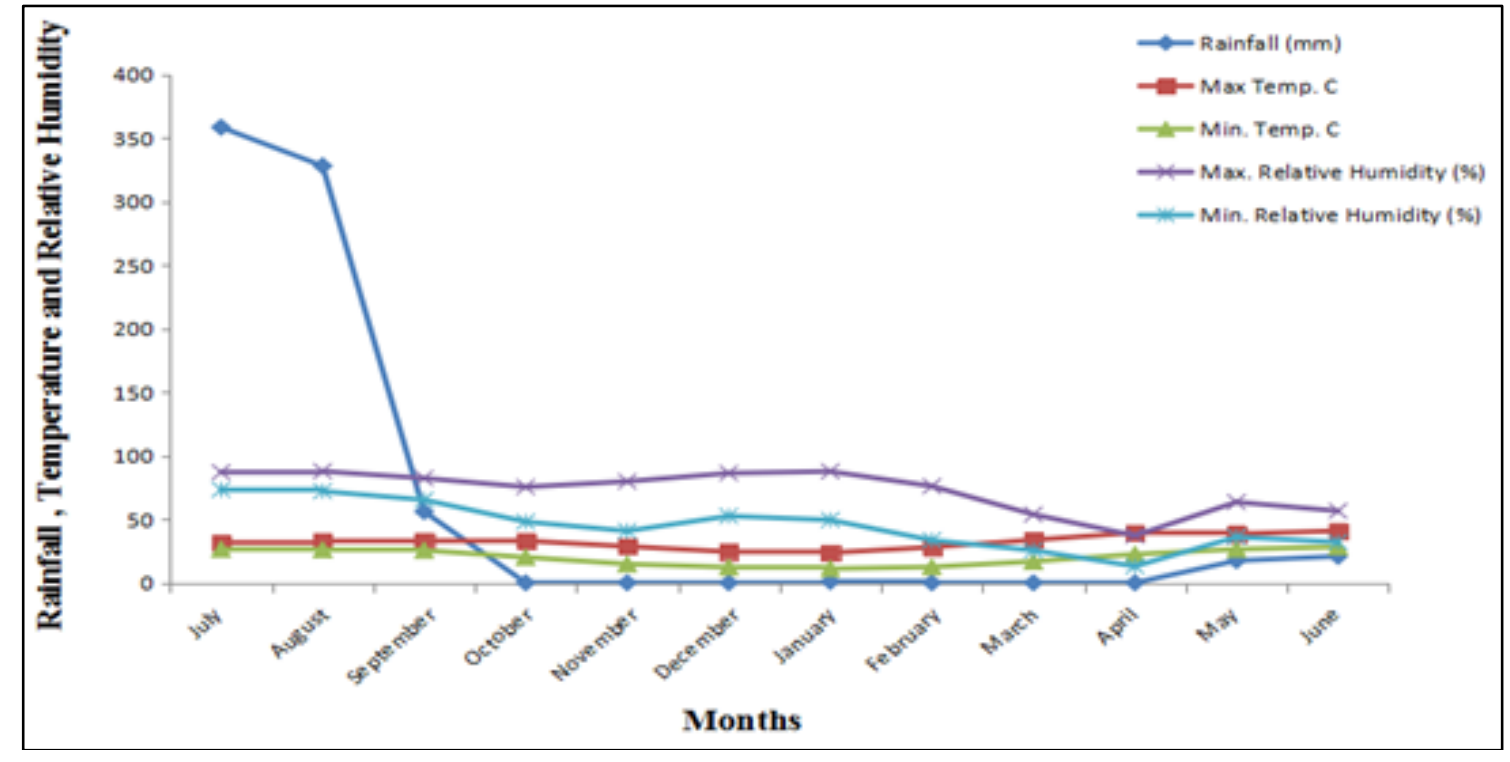

Fig 1: Meteorological data standard (month wise) of Varanasi during 2008-2009.

Isolation of fungi: Fungal populations involved in decomposition of Sesbania aculeata L. green manure were studied and isolated by following methods:

1. Direct observation of green manure samples: The fungi on the decomposing green manure were observed under a binocular microscope (Garrett, 1981) ${ }^{[8]}$.

2. Damp chamber incubation method: In the initial stages of decomposition process, the green manure inhabiting fungi were observed by damp chamber incubation method (Boedijn, 1956) ${ }^{[5]}$. The litter sample was cut into $0.5-1.0 \mathrm{~cm}$ pieces and placed on sterilized blotting paper in Petri-dishes and incubated at $25+2{ }^{\circ} \mathrm{C}$ for 15 days for observation.

3. Dilution plate technique: For the determination of population of mycoflora, their isolation was done by dilution plate technique (Warcup, 1960) ${ }^{[36]}$. In this method, $1 \mathrm{~g}$ green manure sample from the nylon net bag was suspended into $10 \mathrm{ml}$ sterilized distilled water. Further dilution series $\left(1: 10^{3}, 1: 10^{4}, 1: 10^{5}, 1: 10^{6}\right)$ were prepared from it. $1 \mathrm{ml}$ of each dilution was poured individually in sterilized Petri-dishes. Then $15-20 \mathrm{ml}$ of sterilized cool molten potato dextrose agar medium was added. Five replicates of each dilution were prepared and all plates were incubated at $25+2^{\circ} \mathrm{C}$ for a week. The isolated fungi were identified with the help of literature

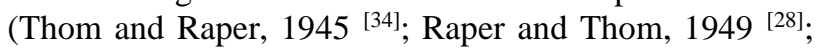

Ellis, 1971 [7]; Subramanium, 1971 [33]; Barnett and

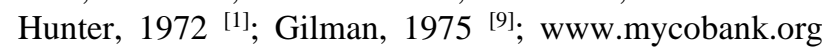
and www.forestryimages.org).

Determination of number of fungi: Total number of fungi/g of oven dried litter (green manure) was calculated using following formula:

$$
\text { No of fungi/g oven dry litter }=\frac{\text { Total number of fungi } \times \text { Concentration of the solution }}{\text { Oven dry weight of the litter }}
$$

Determination of frequency and abundance of fungi: Frequency and abundance of fungi were determined following Saksena $(1955)^{29[]}$.

Frequency: Percent occurrence of a species in the units was calculated in the usual manner and frequency classes were expressed on 1-5 scale as follows:

Class 1: Species occurring in 1-20\% of Petri-dishes;

Class 2: Species occurring in $21-40 \%$ of Petri-dishes;

Class 3: Species occurring in 41-60\% of Petri-dishes;

Class 4: Species occurring in $61-80 \%$ of Petri-dishes;

Class 5: Species occurring in $81-100 \%$ of Petri-dishes.

Abundance: Numerical abundance was calculated from the species on following scale: 
Class 1: Species occurring in 1-3\% of the total species;

Class 2: Species occurring in 4-8\% of the total species;

Class 3: Species occurring in $9-15 \%$ of the total species;

Class 4: Species occurring in $16-25 \%$ of the total species;

Class 5: Species occurring in 26-100\% of the total species.

(B) Decomposition of Sesbania aculeata L. under natural conditions: One set of experimental material Sesbania aculeata L. after cutting at 45 DAS was mixed in the soil and left for decomposition. The decomposing green manure samples were collected at monthly intervals and the $\mathrm{pH}$, moisture content and mycoflora population dynamics (quantitative and qualitative) were estimated by standard methods described above.

Statistical analysis: The data was analyzed using CRD design and result was expressed in terms of LSD (least significant difference).

\section{Results and Discussion}

(A) Decomposition of Sesbania aculeata L. under experimental conditions:

Weight Loss: The weight loss was observed maximum $(30.60 \%)$ in the month of January because of the maximum rainfall in the months of rainy season, which enhanced decomposition process by increased microbial activities. The loss in weight of decomposing green manure was rapid during initial months (Table 1). The process of decay started by the action of decomposers and abiotic environment after the green manure bags were kept in the soil (Kumar et al., 2011) ${ }^{[23]}$. Decomposition results in the change of state of the substrates under the influence of a number of biotic and abiotic factors. Weight loss of substrate has been regarded as the simplest expression of decomposition process which was increased with months perhaps with increased enzymatic production by fungal colonizers (Kumar et al., 2018) [21]. This could be meant that different microbial species have different rates of decomposition as reported by Kumar (2010) ${ }^{[18,24]}$ and Herzog et al. (2019) ${ }^{[10]}$. A small percentage of original weight remaining at last and this may due to there was always litter remaining before new crop (Oladoye et al. 2007) ${ }^{[26]}$.

Table 1: Weight loss of green manure (Sesbania aculeata L.) litter during decomposition

\begin{tabular}{|c|c|c|c|c|c|}
\hline Months & $\begin{array}{l}\text { Dry Weight } \\
\text { of litter (g) }\end{array}$ & \multicolumn{3}{l|}{ Loss of Weight (g) } & \multicolumn{2}{l|}{ Per cent Loss in Weight } \\
\hline & & Monthly & Progressive & Monthly & Progressive*** \\
\hline 2008 & & & & & \\
\hline July & 50.00 & - & - & - & - \\
\hline August & 46.78 & 3.22 & 3.22 & 6.44 & 6.44 \\
\hline September & 37.99 & 8.79 & 12.01 & 18.79 & 24.02 \\
\hline October & 29.78 & 8.21 & 20.22 & 21.61 & 40.44 \\
\hline November & 21.26 & 8.52 & 28.74 & 28.61 & 57.48 \\
\hline December & 16.40 & 4.86 & 33.60 & 22.86 & 67.20 \\
\hline 2009 & & & & & \\
\hline January & 11.38 & 5.02 & 38.62 & 30.60 & 77.24 \\
\hline February & 10.08 & 1.30 & 39.92 & 11.42 & 79.84 \\
\hline March & 8.82 & 1.26 & 41.18 & 12.50 & 82.36 \\
\hline April & 8.15 & 0.67 & 41.85 & 7.59 & 83.70 \\
\hline May & 7.40 & 0.75 & 42.60 & 9.20 & 85.20 \\
\hline June & 6.41 & 0.99 & 43.59 & 13.37 & 87.18 \\
\hline SEm \pm & 0.14 & - & - & 0.01 & 0.01 \\
\hline CD(P=0.05) & 0.42 & - & - & 0.02 & 0.03 \\
\hline Val are
\end{tabular}

Values are mean $(n=3)$

**Additive value of subsequent months

pH: The $\mathrm{pH}$ of decomposing green manure (Sesbania aculeata L.) varied from $5.69 \pm 0.01$ to $7.21 \pm 0.02$ with no definite trend (Fig. 2). The increase in $\mathrm{pH}$ during decomposition was probably due to higher calcium content, rapid loss in citric acid and malic acid through leaching $(\mathrm{Xu}$ and Coventry, 2003; Batty and Younger, 2007) ${ }^{[38,2]}$.

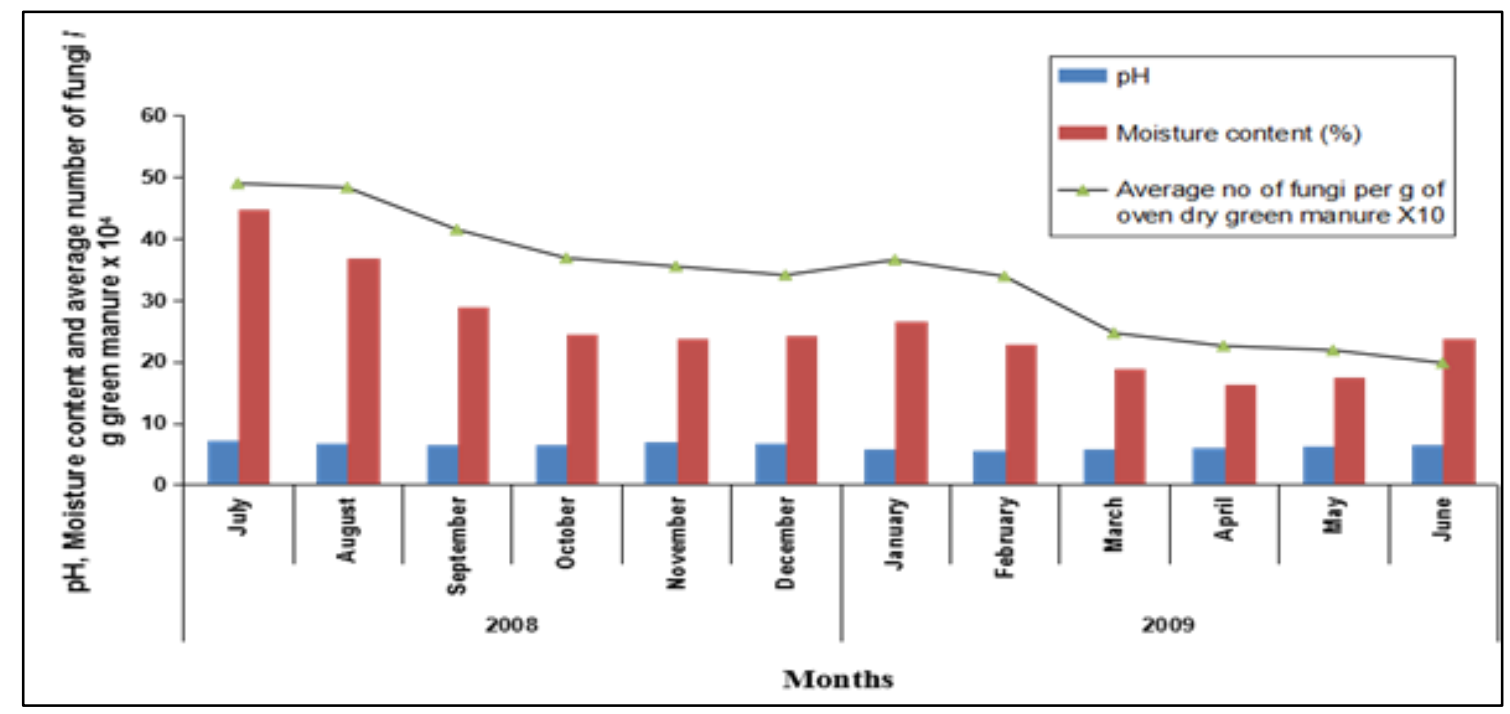

Fig 2: pH, moisture content and average number of fungi per g oven dried decomposing green manure (Sesbania aculeata L.) under experimental conditions

Moisture content: Maximum moisture content was recorded in the month of July $(44.74 \%)$ and thereafter it decreased. The summer months remain almost dry when moisture content gradually decreases in the months of March and April resulting in slower rate of decomposition. The population of fungi in terms of both quantity and quality was found highest in the rainy season. Moisture content is one of the key factors that govern decomposition process (Krishna and Mohan,
2017) ${ }^{[15]}$. High moisture content helps in increasing microbial activities, which could be limited by low moisture content in soil (Manzoni et al., 2012) ${ }^{[25]}$ as moisture content is chiefly responsible for colonization of substrate by microorganisms (Pandey and Sinha, 2008; Kumar, 2010; Krishna and Mohan, 2017) ${ }^{[27,18,24] .}$ 


\section{Population dynamics of Sesbania aculeata L. litter inhabiting mycoflora:}

Quantitative nature of the green manure inhabiting mycoflora: The maximum number $\left(48.95 \times 10^{4}\right)$ of fungi/g litter was recorded in the month of July, whereas minimum was in the month of June $\left(19.78 \times 10^{4}\right)$ (Fig. 2). The average number of fungi involved in the decomposition of Sesbania aculeata $\mathrm{L}$. was found to be dependent on physical climate viz., moisture content, rainfall, temperature and relative humidity. The number of fungi slightly decreased in August and thereafter declined gradually up to December, 2008. After this there was slight increase in the average number of fungi/g of litter in the month of January perhaps due to sporadic rainfall in this month. Thereafter, the fungal population declined heavily up to June. Despite of rainfall in the month of May and June, 2009, the average number of fungi continued to decline may be due to insufficient availability of green manure substrate for further fungal colonization (Kumar, 2010) ${ }^{[18,24]}$.

It was observed in the rainy season, the population of fungi was highest. The decomposition of organic matter started after the residue incorporation (Berkenkamp et al. 2002) ${ }^{[3]}$. Temperature and relative humidity might be responsible for the decline in the number of fungi during summer season. The significance of increasing temperature and decreasing relative humidity of atmosphere as resulted in the decline of fungal population during summer months has also been reported by several workers (Jing et al., 2007; Pandey and Sinha, 2008; Kumar et al., 2013) ${ }^{[27,12,16]}$.

The higher number of the fungi/g of substrate in initial stages of decomposition was due to availability of the maximum substrate residue in the soil, whereas the fungal population declined due to physiological constraints in the later stage of decomposition (Sinha et al., 2010) ${ }^{[32]}$. While on the other hand favourable climatic conditions viz. high relative humidity, moderate temperature and high moisture content of decaying tissues also favoured the better colonization during rainy season.

During the winter season the colonization found to be in declining order because the temperature below optimum level does not favour sporulation of fungi (Jing et al., 2007; Kumar, $2010)^{[12,18,24]}$. The minimum number of fungi was found in the end of experimental period may be due to unavailability of the substrate. Same pattern of fungal succession was also reported by several workers (Pandey and Sinha, 2008; Wardle et al., 2009; Krishna and Mohan, 2017) ${ }^{[27,37,15] .}$

Qualitative nature of the green manure inhabiting mycoflora: Green manures are initially colonized by various saprophytic and weak parasitic fungal species before they are incorporated in the soil and further colonization of litter by fungi depends on the climatic conditions and the nature of substrate (Pandey and Sinha, 2008) ${ }^{[27]}$. The fungal species were divided into early and late colonizers according to their occurrence during experiment. Different techniques viz., direct observation method, damp chamber incubation method and dilution plate technique were used for the isolation with the expectation that they would reduce the bias associated with single technique.

Direct observation method: Sixteen fungal species were observed directly under the binocular microscope. Out of these, Aspergillus niger, A. fumigatus, Penicillium citrinum, Trichoderma harzianum, Alternaria alternata, Curvularia lunata, Cladosporium cladosporioides, Fusarium semitectum and dark sterile mycelium were observed as dominant fungi (Table 2).

Table 2: Direct observation of fungal species from decomposing Sesbania aculeata L. by binocular microscope under experimental conditions

\begin{tabular}{|c|c|c|c|c|c|c|c|c|c|c|c|c|}
\hline \multirow[t]{2}{*}{ Fungal Species } & \multicolumn{6}{|c|}{2008} & \multicolumn{6}{|c|}{2009} \\
\hline & July & Aug & Sept & Oct & Nov & Dec & Jan & Feb & Mar & Apr & May & Jun \\
\hline Rhizopus stolonifer & - & + & - & + & - & - & - & - & - & - & - & - \\
\hline Choanephora cucurbitarum & + & + & - & - & - & - & - & - & - & - & - & - \\
\hline Colletotrichum falcatum & + & - & - & - & - & - & - & - & - & - & - & - \\
\hline Aspergillus fumigatus & + & + & + & - & + & - & - & + & - & - & - & - \\
\hline Aspergillus niger & + & + & + & + & + & + & + & + & + & + & + & + \\
\hline Penicillium citrinum & + & + & + & - & + & + & + & + & - & + & + & + \\
\hline Trichoderma harzianum & - & + & + & + & + & + & + & + & - & + & + & + \\
\hline Alternaria alternata & - & - & + & + & + & + & + & + & - & + & - & - \\
\hline Curvularia lunata & - & - & + & + & - & + & - & + & + & + & + & + \\
\hline Humicola grisea & - & - & - & - & - & - & - & + & + & - & - & + \\
\hline Nigrospora sphaerica & - & - & - & + & + & - & - & - & - & - & - & - \\
\hline Torula graminis & + & - & - & - & - & - & - & - & + & - & - & - \\
\hline Cladosporium cladosporioides & - & - & + & + & + & + & + & + & + & - & + & + \\
\hline Epicoccum purpurascens & + & - & - & - & + & - & - & - & - & - & - & - \\
\hline Fusarium semitectum & + & + & + & - & + & + & + & + & + & + & - & + \\
\hline Dark sterile mycelium & + & + & - & + & + & + & + & + & + & + & - & + \\
\hline
\end{tabular}

$+=$ Present, - = Absent

Damp chamber incubation method: By this method sampling was performed from July 2008 to September 2008 because the green manure was broken down into small pieces after this period which was very difficult to handle for sampling. A total number of 23 fungal species were isolated by this method (Table 3). Aspergillus flavus, A. niger, Penicillium citrinum, Trichoderma harzianum, Alternaria alternata, Curvularia lunata, Cladosporium cladosporioides, Fusarium semitectum and dark sterile mycelium were recorded as dominant species, whereas Mortierella subtillissima, Phoma hibernica, Drechslera avenacea, Nigrospora sphaerica, Epicoccum purpurascens and pink sterile mycelium were observed only once by this method. 
Table 3: Fungi recorded from decomposing Sesbania aculeata L. by damp chamber incubation method under experimental conditions

\begin{tabular}{|c|c|c|c|}
\hline \multirow[t]{2}{*}{ Fungal Species } & \multicolumn{3}{|c|}{ Months } \\
\hline & July & August & September \\
\hline Pythium aphanidermatum & + & + & - \\
\hline Rhizopus stolonifer & + & + & - \\
\hline Mucor racemosus & + & + & - \\
\hline Mortierella subtilissima & + & - & - \\
\hline Chaetomium globosum & + & + & - \\
\hline Phoma hibernica & - & - & + \\
\hline Pestalotia mangiferae & + & - & + \\
\hline Aspergillus flavus & + & + & + \\
\hline Aspergillus niger & + & + & + \\
\hline Penicillium citrinum & + & + & + \\
\hline Trichoderma harzianum & + & + & + \\
\hline Alternaria alternata & + & + & + \\
\hline Curvularia lunata & + & + & + \\
\hline Drechslera avenacea & - & - & + \\
\hline Nigrospora sphaerica & - & - & + \\
\hline Torula graminis & + & - & + \\
\hline Cladosporium cladosporioides & + & + & + \\
\hline Epicoccum purpurascens & + & - & - \\
\hline Fusarium semitectum & + & + & + \\
\hline Fusarium moniliforme & + & + & - \\
\hline Dark sterile mycelium & + & + & + \\
\hline Pink sterile mycelium & - & + & - \\
\hline Unidentified & + & - & - \\
\hline
\end{tabular}

+=Present, - = Absent

Dilution plate technique: A total number of 42 fungal species was isolated by this method (Table 4). Aspergillus luchuensis, A. candidus, A. sydowi, A. sulphureus, A. terreus, Penicillium rubrum, $P$. chrysogenum, Trichoderma viride, $T$. koningii, Gliocladium roseum, Alternaria solani, Curvularia pallenscens, Diplococcum spicatum, Helminthosporium oyzae, Fusarium solani, Myrothecium roridum and white sterile mycelium were observed by only this technique.

The diverse fungal population appeared at different stages of decomposition may be classified into four different groups depending upon their occurrence during experimental period.

Table 4: Frequency and Abundance of mycoflora associated with the decomposing Sesbania aculeata L. under experimental conditions

\begin{tabular}{|c|c|c|c|c|c|c|c|c|c|c|c|c|c|c|c|c|c|c|c|c|c|c|c|c|c|c|}
\hline \multirow{3}{*}{ Fungal Species } & \multicolumn{13}{|c|}{2008} & \multicolumn{13}{|c|}{2009} \\
\hline & \multicolumn{2}{|c|}{ Jul } & \multicolumn{2}{|c|}{ Aug } & \multicolumn{2}{|c|}{ Sept } & \multicolumn{2}{|c|}{ Oct } & \multicolumn{2}{|c|}{ Nov } & \multicolumn{3}{|c|}{ Dec } & \multicolumn{3}{|c|}{ Jan } & \multicolumn{2}{|c|}{ Feb } & \multicolumn{2}{|c|}{ Mar } & \multicolumn{2}{|c|}{ Apr } & \multicolumn{2}{|c|}{ May } & \multicolumn{2}{|c|}{ Jun } \\
\hline & & $\bar{A}$ & $\mathbf{F}$ & $\mathbf{A}$ & $\mathbf{F}$ & $\mathbf{A}$ & $\mathbf{F}$ & $\mathbf{A}$ & $\mathbf{F}$ & $\mathbf{A}$ & & & $\mathbf{A}$ & $\mathbf{F}$ & \begin{tabular}{l|l}
$\mathbf{F}$ & $\mathbf{A}$ \\
\end{tabular} & $\begin{array}{ll}\mathbf{A} & \mathbf{F} \\
\end{array}$ & \begin{tabular}{l|l}
$\mathbf{F}$ & \\
\end{tabular} & $\mathbf{A}$ & $\mathbf{F}$ & $\mathbf{A}$ & $\mathbf{F}$ & $\mathbf{A}$ & $\mathbf{F}$ & $\mathbf{A}$ & $\mathbf{F}$ & $\mathbf{A}$ \\
\hline Pythium aphanidermatum & 2 & 1 & 3 & 2 & - & - & - & - & - & - & & & - & - & $-1-$ & $-1-$ & - & - & - & - & - & - & - & - & - & - \\
\hline Rhizopus stolonifer & 4 & 3 & 3 & 4 & 3 & 2 & 2 & 1 & - & - & & & - & 5 & \begin{tabular}{l|l}
5 & 4
\end{tabular} & 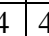 & 4 & 3 & - & - & - & - & 2 & 2 & - & - \\
\hline Mucor racemosus & 2 & 2 & 4 & 3 & - & - & - & - & 4 & 3 & & & 3 & - & - & -- & - & - & - & - & - & - & - & - & - & - \\
\hline Chaetomium globosum & - & - & - & - & - & - & 2 & 2 & 1 & 1 & & & - & - & -- & -- & - & - & - & - & - & - & - & - & - & - \\
\hline Phoma hibernica & - & - & - & - & 1 & 1 & 2 & 2 & 3 & 2 & & & - & 2 & $2 \mid 3$ & 33 & 3 & 3 & - & - & - & - & - & - & - & - \\
\hline Macrophomina phaseoli & - & - & - & - & - & - & - & - & - & - & & & - & - & -- & -1 & 1 & 1 & 3 & 3 & 3 & 2 & - & - & - & - \\
\hline Pestalotia mangiferae & 1 & 1 & 1 & 1 & 2 & 1 & - & - & 2 & 1 & & 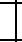 & 1 & - & -- & -- & - & - & - & - & - & - & - & - & - & - \\
\hline Aspergillus fumigatus & 5 & 5 & 4 & 5 & 3 & 4 & 5 & 5 & 4 & 3 & & & - & 3 & \begin{tabular}{l|l}
3 & 3 \\
\end{tabular} & \begin{tabular}{l|l}
3 & 1 \\
\end{tabular} & 1 & 1 & - & - & - & - & - & - & - & - \\
\hline Aspergillus flavus & 5 & 3 & 5 & 4 & 2 & 3 & 1 & 1 & 3 & 3 & & & 3 & 4 & \begin{tabular}{l|l}
4 & 3 \\
\end{tabular} & 32 & 2 & 1 & 1 & 2 & 3 & 4 & 2 & 1 & 1 & 1 \\
\hline Aspergillus niger & 4 & 4 & 4 & 5 & 5 & 5 & 5 & 4 & 5 & 3 & & & 2 & 4 & \begin{tabular}{l|l}
4 & 3 \\
\end{tabular} & 32 & 2 & 2 & 4 & 5 & 2 & 2 & 1 & 2 & 2 & 1 \\
\hline Aspergillus luchuensis & - & - & - & - & - & - & - & - & - & - & & & 2 & - & - & -- & - & - & - & - & 2 & 2 & - & - & - & - \\
\hline Aspergillus candidus & - & - & - & - & 1 & 1 & - & - & - & - & & & 1 & 2 & 22 & $2-$ & - & - & 1 & 1 & - & - & - & - & - & - \\
\hline Aspergillus sydowi & - & - & - & - & 3 & 2 & 1 & 1 & - & - & & & - & - & -- & -- & - & - & - & - & - & - & - & - & - & - \\
\hline Aspergillus sulphureus & 1 & 1 & - & - & - & - & - & - & 1 & 1 & & & - & 2 & \begin{tabular}{l|l}
2 & 1 \\
\end{tabular} & 12 & 2 & 2 & 2 & 2 & - & - & - & - & - & - \\
\hline Aspergillus terreus & - & - & 1 & 1 & - & - & 2 & 1 & - & - & & & 2 & 3 & $\begin{array}{ll}3 & 2 \\
\end{array}$ & 22 & 2 & 2 & - & - & - & - & - & - & - & - \\
\hline Penicillium rubrum & - & - & 1 & 1 & 3 & 2 & 5 & 3 & 2 & 1 & & & 4 & 4 & $\begin{array}{ll}4 & 3 \\
\end{array}$ & 33 & 3 & 5 & 5 & 5 & 2 & 2 & 3 & 1 & 1 & 1 \\
\hline Penicillium citrinum & 5 & 3 & 4 & 2 & 3 & 2 & 5 & 5 & 2 & 3 & & & 3 & 5 & 5 & \begin{tabular}{l|l}
5 & 4
\end{tabular} & 4 & 2 & 5 & 3 & 5 & 4 & 2 & 2 & 1 & 1 \\
\hline Penicillium chrysogenum & - & - & - & - & - & - & - & - & - & - & & & 1 & - & - & -- & - & - & - & - & - & - & - & - & - & - \\
\hline Trichoderma harzianum & 1 & 1 & 1 & 1 & 2 & 2 & 1 & 1 & 4 & 3 & & & 4 & 5 & 55 & \begin{tabular}{l|l}
5 & 4 \\
\end{tabular} & 4 & 2 & 2 & 3 & 5 & 2 & 2 & 1 & 1 & 1 \\
\hline Trichoderma viride & - & - & - & - & - & - & - & - & - & - & & & 2 & - & - & -1 & 1 & 1 & 1 & 3 & 1 & 1 & 1 & 1 & - & - \\
\hline Trichoderma koningii & - & - & - & - & 4 & 3 & 4 & 5 & 3 & 2 & & & - & - & - & -2 & 2 & 2 & - & - & - & - & - & - & - & - \\
\hline Gliocladium roseum & - & - & - & - & 4 & 3 & 2 & 2 & 3 & 4 & & & - & - & - & -2 & 2 & 2 & - & - & - & - & - & - & - & - \\
\hline Alternaria alternata & - & - & - & - & 3 & 2 & 2 & 1 & 1 & 1 & & & 2 & 2 & 2 & $1 \quad 1$ & 1 & 1 & - & - & 2 & 2 & - & - & - & - \\
\hline Alternaria solani & - & - & - & - & 1 & 2 & 2 & 1 & - & - & & & - & - & - & -- & - & - & - & - & - & - & - & - & - & - \\
\hline Curvularia lunata & - & - & - & - & 1 & 1 & 2 & 2 & - & - & & & 1 & - & - & -2 & 2 & 3 & 5 & 4 & 5 & 5 & 3 & 4 & 5 & 2 \\
\hline Curvularia pallescens & 1 & 1 & 1 & 1 & - & - & - & - & 1 & 2 & & & 2 & - & - & -- & - & - & - & - & - & - & - & - & - & - \\
\hline Drechslera avenacea & 2 & 1 & 3 & 2 & 3 & 2 & 2 & 2 & - & - & & & - & - & - & -1 & 1 & 1 & 2 & 2 & - & - & - & - & - & - \\
\hline Humicola grisea & - & - & - & - & - & - & - & - & 1 & 1 & & & - & 2 & 2 & $2-$ & - & - & - & - & - & - & - & - & - & - \\
\hline Nigrospora sphaerica & - & - & - & - & - & - & - & - & - & - & & & 2 & - & - & -- & - & - & - & - & 1 & 1 & - & - & -1 & - \\
\hline
\end{tabular}




\begin{tabular}{|c|c|c|c|c|c|c|c|c|c|c|c|c|c|c|c|c|c|c|c|c|c|c|c|c|}
\hline Torula graminis & 1 & 1 & 2 & 2 & 1 & 1 & - & - & - & - & - & - & - & - & - & - & 1 & 1 & 1 & 1 & - & - & - & - \\
\hline Diplococcium spicatum & 1 & 1 & 1 & 1 & - & - & - & - & - & - & - & - & - & - & - & - & - & - & - & - & - & - & - & - \\
\hline Helminthosporium oryzae & 1 & 1 & 1 & 1 & 2 & 1 & - & - & - & - & - & - & - & - & 1 & 1 & - & - & - & - & 1 & 1 & - & - \\
\hline Cladosporium cladosporioides & - & - & - & - & - & - & - & - & 5 & 5 & 5 & 5 & 4 & 5 & 4 & 4 & 4 & 3 & 3 & 2 & 1 & 1 & - & - \\
\hline Epicoccum purpurascens & 2 & 2 & 1 & 1 & - & - & 1 & 1 & - & - & - & - & - & - & - & - & - & - & - & - & - & - & - & - \\
\hline Fusarium semitectum & 5 & 5 & 5 & 4 & 5 & 3 & 5 & 4 & 4 & 2 & 2 & 3 & 1 & 1 & - & - & 2 & 2 & 5 & 4 & - & - & - & - \\
\hline Fusarium solani & - & - & 2 & 2 & 1 & 1 & - & - & - & - & 3 & 4 & 5 & 4 & - & - & - & - & - & - & 1 & 1 & - & - \\
\hline Fusarium moniliforme & - & - & 2 & 1 & 1 & 1 & 3 & 2 & 3 & 2 & 1 & 1 & - & - & 1 & 1 & - & - & - & - & - & - & - & - \\
\hline Myrothecium roridum & - & - & - & - & 1 & 1 & - & - & - & - & - & - & - & - & - & - & - & - & - & - & - & - & - & - \\
\hline Dark sterile mycelium & 5 & 4 & 3 & 2 & 5 & 4 & - & - & 5 & 3 & 5 & 2 & 3 & 4 & 3 & 5 & - & - & 2 & 2 & 1 & 1 & - & - \\
\hline White sterile mycelium & - & - & - & - & 2 & 2 & - & - & 3 & 2 & - & - & - & - & 2 & 2 & - & - & 1 & 1 & - & - & - & - \\
\hline Pink sterile mycelium & - & - & - & - & - & - & - & - & - & - & - & - & 1 & 1 & - & - & - & - & 1 & 1 & - & - & - & - \\
\hline Unidentified & 1 & 1 & - & - & - & - & - & - & - & - & - & - & - & - & 1 & 1 & - & - & - & - & - & - & - & - \\
\hline
\end{tabular}

$\mathrm{F}=$ Frequency, $\mathrm{A}=$ Abundance

Group I: Dominant Fungi: The fungi which appeared in more than six months during isolation period were included in this group. Rhizopus stolonifer, Aspergillus fumigatus, A. flavus, A. niger, Penicillium rubrum, P. citrinum, Trichoderma harzianum, Alternaria alternata, Curvularia lunata, Cladosporium cladosporioides, Fusarium semitectum and dark sterile mycelium were found as dominant fungi.

Group II: Common Fungi: The group of common fungi occurred in five to six months of experimental period with less frequency and abundance than the dominant ones. The common fungi isolated were Phoma hibernica, Pestolotia mangiferae, Aspergillus sulphureus, A. terreus, Trichoderma viride, Drechslera avenacea, Torula graminis, Fusarium solani and $F$. moniliforme.

Group III: Frequent Fungi: This group includes the mycoflora which appeared in at least three to four months of experimental period with normal frequency and abundance. Mucor racemosus, Aspergillus candidus, Trichoderma koningii, Gliocladium roseum, Curvularia pallenscens and white sterile mycelium were found to be frequent fungi.
Group IV: Rare Fungi: The fungi of this group appeared only once or twice with very low frequency and abundance. Pythium aphanidermatum, Chaetomium globosum, Aspergillus luchuensis, A. sydowi, Penicillium chrysogenum, Alternaria solani, Humicola grisea, Nigrospora sphaerica, Diplococcum spicatum, Myrothecium roridum and pink sterile mycelium were recorded as member of this group.

(B). Decomposition of Sesbania aculeata L. mixed in soil under natural conditions

pH: The $\mathrm{pH}$ of decomposing green manure (Sesbania aculeata L.) litter mixed in soil varied from $6.70 \pm 0.20$ to $7.20 \pm 0.70$ with no definite trend (Table 5). The increase in $\mathrm{pH}$ during decomposition was probably due to higher calcium content, rapid loss in citric acid and malic acid through leaching (Xu and Coventry, 2003; Batty and Younger, 2007) $[38,2]$.

Moisture content: Maximum moisture content was recorded in the month of July $(46.20 \%)$ and thereafter it decreased (Table 5). Moisture content is one of the key factors that govern decomposition process (Krishna and Mohan, 2017) [15].

Table 5: $\mathrm{pH}$, moisture content and average number of fungi per g oven dry decomposing green manure (Sesbania aculeata L.) under natural conditions.

\begin{tabular}{|c|c|c|c|}
\hline \multicolumn{5}{|c|}{$\mathbf{p H}$} & Moisture content (\%) & Average no of fungi per G of oven dry green manure X10 $^{\mathbf{4}}$ \\
\hline Months & \multicolumn{3}{|c|}{$\mathbf{2 0 0 8}$} \\
\hline July & $6.70+0.02$ & $46.20+0.30$ & $47.85+0.20$ \\
\hline August & $6.90+0.05$ & $38.76+0.85$ & $46.96+0.94$ \\
\hline September & $7.18+0.10$ & $29.72+0.25$ & $40.84+0.15$ \\
\hline October & $6.88+0.06$ & $26.40+0.95$ & $37.50+0.56$ \\
\hline November & $6.85+0.09$ & $23.45+0.20$ & $36.64+0.10$ \\
\hline December & $7.15+0.12$ & $23.80+0.64$ & $34.86+0.20$ \\
\hline \multicolumn{5}{|c|}{$\mathbf{2 0 0 9}$} \\
\hline January & $7.00+0.15$ & $27.18+0.25$ & $35.54+0.02$ \\
\hline February & $7.20+0.70$ & $22.50+0.90$ & $32.68+0.26$ \\
\hline March & $6.82+0.02$ & $17.58+0.46$ & $23.80+0.30$ \\
\hline April & $6.90+0.17$ & $15.98+0.65$ & $21.90+0.16$ \\
\hline May & $7.00+0.04$ & $17.85+0.34$ & $20.48+0.45$ \\
\hline June & $7.10+0.06$ & $24.20+0.40$ & $21.32+0.20$ \\
\hline
\end{tabular}

Values are mean $(n=3)+S D$

Population dynamics of Sesbania aculeata L. litter (mixed in soil) inhabiting mycoflora:

Quantitative nature of green manure inhabiting mycoflora: The maximum fungal population $\left(47.85 \times 10^{4}\right)$ per gram decomposing dry litter incorporated in the field soil was in the month of July, 2008 (Table 5), approximately after 30 days of litter amendment in soil during decomposition period. The minimum fungal population was recorded in the month of May, $2009\left(20.48 \times 10^{4}\right)$.
The dynamics of fungal population can be attributed to abiotic variables viz., temperature, moisture content, type of soil and nature of the substrate (Kumar, 2010) ${ }^{[18,24]}$.

Qualitative nature of green manure litter inhabiting mycoflora: The mycoflora were classified into four groups depending upon frequency, abundance, sporulation and time of appearance in different months. 
Direct observation method: Eighteen fungal species were observed directly under the binocular microscope (Table 6). Out of these, Aspergillus niger, Penicillium citrinum,
Trichoderma harzianum, Alternaria alternata, Curvularia lunata, Cladosporium cladosporioides, Fusarium semitectum and dark sterile mycelium were observed as dominant fungi.

Table 6: Direct observation of fungal species from decomposing Sesbania aculeata L. by binocular microscope under natural conditions

\begin{tabular}{|c|c|c|c|c|}
\hline \multirow{2}{*}{ Fungal Species } & \multicolumn{4}{|c|}{2008} \\
\hline & July & Aug & Sept & Oct \\
\hline Rhizopus stolonifer & - & + & - & + \\
\hline Choanephora cucurbitarum & + & + & - & - \\
\hline Colletotrichum falcatum & + & - & - & - \\
\hline Aspergillus fumigatus & + & - & + & - \\
\hline Aspergillus niger & + & + & + & + \\
\hline Penicillium citrinum & + & + & + & + \\
\hline Trichoderma harzianum & - & + & + & + \\
\hline Alternaria alternata & + & - & + & + \\
\hline Curvularia lunata & + & - & + & + \\
\hline Humicola grisea & - & - & - & + \\
\hline Nigrospora sphaerica & - & - & - & + \\
\hline Torula graminis & + & - & - & - \\
\hline Cladosporium cladosporioides & - & + & + & + \\
\hline Epicoccum purpurascens & + & + & - & - \\
\hline Fusarium semitectum & + & + & + & - \\
\hline Drechslera avanacea & + & + & - & - \\
\hline Diplococcum spicatum & + & - & - & + \\
\hline Dark sterile mycelium & + & + & - & + \\
\hline
\end{tabular}

Damp chamber incubation method: By this method sampling was performed from July 2008 to September 2008 because the green manure was broken down into small pieces after this period which was very difficult to handle for sampling. A total number of 21 fungal species were isolated by this method (Table 7). Pythium aphanidermatum, Aspergillus flavus, A. niger, Penicillium citrinum,
Trichoderma harzianum, Curvularia lunata, Cladosporium cladosporioides, Fusarium semitectum and dark sterile mycelium were recorded as dominant species, whereas Phoma hibernica, Drechslera avenacea, Humicola grisea and pink sterile mycelium were observed only once by this method.

Table 7: Fungi recorded from Sesbania aculeata L. litter by damp chamber incubation method under natural conditions

\begin{tabular}{|c|c|c|c|}
\hline \multirow[t]{2}{*}{ Fungal Species } & \multicolumn{3}{|c|}{ Months } \\
\hline & July & August & September \\
\hline Pythium aphanidermatum & + & + & + \\
\hline Rhizopus stolonifer & + & + & - \\
\hline Chaetomium globosum & + & + & - \\
\hline Phoma hibernica & - & + & - \\
\hline Pestalotia mangiferae & + & - & + \\
\hline Aspergillus flavus & + & + & + \\
\hline Aspergillus niger & + & + & + \\
\hline Aspergillus sulphureus & + & + & - \\
\hline Penicillium citrinum & + & + & + \\
\hline Trichoderma harzianum & + & + & + \\
\hline Alternaria alternata & - & + & + \\
\hline Curvularia lunata & + & + & + \\
\hline Drechslera avenacea & - & - & + \\
\hline Diplococcium spicatum & + & - & - \\
\hline Torula graminis & + & - & + \\
\hline Cladosporium cladosporioides & + & + & + \\
\hline Humicola grisea & + & - & - \\
\hline Fusarium semitectum & + & + & + \\
\hline Fusarium moniliforme & + & + & - \\
\hline Dark sterile mycelium & + & + & + \\
\hline Pink sterile mycelium & - & - & + \\
\hline
\end{tabular}

+=Present, - = Absent

Dilution plate technique: A total number of 37 fungal species were isolated by this method (Table 8). Mucor racemosus, Phoma glomerata, Macrophomina phaseoli, Aspergillus luchuensis, A. sydowi, A. terreus, Penicillium rubrum, Trichoderma viride, T. koningii, Gliocladium roseum, Alternaria solani, Fusarium solani, Myrothecium roridum and white sterile mycelium were observed by only this technique.

The diverse fungal population appeared at different stages of decomposition under natural conditions may be classified into four different groups depending upon their occurrence during experimental period. 
Table 8: Frequency and Abundance of fungal species associated with the decomposing Sesbania aculeata L. litter mixed in soil under natural conditions

\begin{tabular}{|c|c|c|c|c|c|c|c|c|c|c|c|c|c|c|c|c|c|c|c|c|c|c|c|c|c|}
\hline \multirow{3}{*}{ Fungal Species } & \multicolumn{12}{|c|}{2008} & & \multicolumn{12}{|c|}{2009} \\
\hline & \multicolumn{2}{|c|}{ Jul } & \multicolumn{2}{|c|}{ Aug } & \multicolumn{2}{|c|}{ Sept } & \multicolumn{2}{|c|}{ Oct } & \multicolumn{2}{|c|}{ Nov } & \multicolumn{2}{|c|}{ Dec } & \multicolumn{3}{|c|}{ Jan } & \multicolumn{2}{|c|}{ Feb } & \multicolumn{2}{|c|}{ Mar } & \multicolumn{2}{|c|}{ Apr } & \multicolumn{2}{|c|}{ May } & \multicolumn{2}{|c|}{ Jun } \\
\hline & $\mathbf{F}$ & $\mathbf{A}$ & $\mathbf{F}$ & $\mathbf{A}$ & $\mathbf{F}$ & $\mathbf{A}$ & $\mathbf{F}$ & $\mathbf{A}$ & $\mathbf{F}$ & $\mathbf{A}$ & $\mathbf{F}$ & $\mathbf{A}$ & $\mathbf{F}$ & & $\mathbf{A}$ & $\mathbf{F}$ & $\mathbf{A}$ & $\mathbf{F}$ & $\mathbf{A}$ & $\mathbf{F}$ & $\mathbf{A}$ & $\mathbf{F}$ & $\mathbf{A}$ & $\mathbf{F}$ & $\mathbf{A}$ \\
\hline Pythium aphanidermatum & - & - & 1 & 1 & - & - & 1 & 1 & - & - & - & - & - & & - & - & - & - & - & - & - & - & - & - & - \\
\hline Rhizopus stolonifer & 2 & 4 & 3 & 4 & 3 & 2 & - & - & - & - & - & - & 5 & 5 & 4 & 4 & 3 & - & - & - & - & 1 & 1 & 1 & 1 \\
\hline Mucor racemosus & - & - & 2 & 1 & 2 & 3 & - & - & - & - & 1 & 2 & 2 & 2 & 3 & - & - & - & - & - & - & - & - & - & - \\
\hline Chaetomium globosum & - & - & - & - & 2 & 3 & 2 & 2 & 1 & 1 & - & - & - & 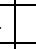 & - & - & - & - & - & 1 & 1 & - & - & - & - \\
\hline Phoma glomerata & 5 & 3 & 3 & 1 & - & - & 3 & 4 & 5 & 4 & 2 & 4 & 2 & 2 & 2 & 4 & 3 & 1 & 1 & - & - & - & - & - & - \\
\hline Macrophomina phaseoli & - & - & - & - & - & - & - & - & - & - & - & - & 1 & & 2 & 1 & 1 & 1 & 2 & - & - & - & - & - & - \\
\hline Pestalotia mangiferae & - & - & - & - & 2 & 1 & - & - & 1 & 1 & - & - & 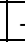 & & - & - & - & - & - & - & - & 1 & 1 & - & - \\
\hline Aspergillus flavus & 2 & 3 & 4 & 3 & 5 & 4 & 2 & 3 & 1 & 1 & 3 & 2 & 14 & 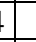 & 3 & 4 & 2 & 2 & 1 & 1 & 2 & - & - & - & - \\
\hline Aspergillus fumigatus & 5 & 4 & 4 & 5 & 3 & 4 & 5 & 2 & 4 & 2 & - & - & 2 & 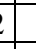 & 1 & 1 & 1 & - & - & - & - & 1 & 1 & - & - \\
\hline Aspergillus niger & 4 & 3 & 4 & 5 & 5 & 2 & 5 & 5 & 4 & 5 & 3 & 2 & 14 & 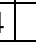 & 1 & 2 & 2 & 4 & 3 & 2 & 1 & - & - & - & - \\
\hline Aspergillus luchuensis & - & - & - & - & - & - & - & - & - & - & 4 & 1 & - & & - & - & - & - & - & 2 & 1 & - & - & - & - \\
\hline Aspergillus sydowi & - & - & - & - & 4 & 2 & 1 & 1 & - & - & 1 & 2 & 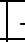 & & - & - & - & - & - & - & - & - & - & - & - \\
\hline Aspergillus sulphurous & 1 & 1 & 1 & 2 & - & - & - & - & 1 & 1 & 2 & 1 & 2 & & 3 & 2 & 1 & - & - & - & - & 1 & 1 & - & - \\
\hline Aspergillus terreus & - & - & 1 & 1 & - & - & - & - & 2 & 1 & 1 & 1 & 3 & 3 & 2 & 4 & 3 & - & - & 1 & 1 & - & - & - & - \\
\hline Penicillium citrinum & 5 & 4 & 4 & 2 & 3 & 2 & 5 & 4 & 3 & 2 & 3 & 4 & 14 & & 2 & 3 & 1 & 5 & 3 & 5 & 4 & 2 & 1 & 1 & 1 \\
\hline Penicillium rubrum & 2 & 3 & 2 & 1 & - & - & 1 & 1 & 3 & 2 & 5 & 4 & 2 & & 3 & 4 & 3 & 1 & 2 & - & - & - & - & 1 & 3 \\
\hline Trichoderma harzianum & - & - & 1 & 1 & 1 & 2 & 3 & 2 & 4 & 3 & 5 & 3 & 14 & 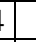 & 2 & 4 & 5 & 2 & 1 & 2 & 4 & 2 & 1 & 1 & 1 \\
\hline Trichoderma koningii & 1 & 1 & - & - & - & - & 3 & 4 & 4 & 5 & 3 & 2 & 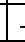 & 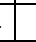 & - & - & - & 2 & 1 & 3 & 2 & - & - & - & - \\
\hline Gliocladium roseum & 2 & 3 & 4 & 2 & 5 & 4 & 1 & 2 & 1 & 1 & - & - & 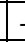 & & - & 2 & 1 & 3 & 2 & 4 & 5 & - & - & - & - \\
\hline Alternaria alternata & - & - & - & - & 3 & 2 & 2 & 1 & 1 & 1 & 3 & 2 & 2 & & 1 & 1 & 1 & - & - & 2 & 3 & - & - & - & - \\
\hline Alternaria solani & - & - & - & - & 4 & 3 & - & - & 1 & 2 & 2 & 1 & 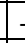 & 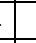 & - & - & - & - & - & - & - & - & - & - & - \\
\hline Curvularia lunata & 5 & 3 & 5 & 2 & - & - & 1 & 3 & 4 & 2 & - & - & 1 & & 1 & - & - & 2 & 3 & 5 & 4 & 5 & 5 & 3 & 4 \\
\hline Drechslera avenacea & 2 & 1 & 3 & 2 & 3 & 1 & 2 & 1 & - & - & - & - & 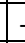 & & - & 1 & 2 & 2 & 3 & - & - & - & - & - & - \\
\hline Humicola grisea & - & - & - & - & - & - & 1 & 1 & - & - & 1 & 2 & - & & - & - & - & - & - & - & - & - & - & - & - \\
\hline Nigrospora sphaerica & - & - & - & - & - & - & - & - & - & - & - & - & 2 & 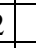 & 1 & 1 & 1 & - & - & - & - & - & - & - & - \\
\hline Torula graminis & 1 & 2 & 2 & 2 & 1 & 1 & - & - & - & - & - & - & 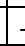 & . & - & - & - & 1 & 1 & 1 & 1 & - & - & - & - \\
\hline Diplococcium spicatum & 1 & 2 & 1 & 1 & - & - & - & - & - & - & - & - & - & & - & - & - & - & - & - & - & - & - & - & - \\
\hline Cladosporium cladosporioides & - & - & - & - & - & - & 5 & 4 & 5 & 3 & 4 & 5 & 4 & 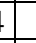 & 4 & 3 & 2 & 3 & 1 & 1 & 1 & - & - & - & - \\
\hline Epicoccum purpurascens & 2 & 1 & 1 & 2 & - & - & 1 & 1 & - & - & - & - & - & 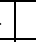 & - & - & - & - & - & - & - & - & - & - & - \\
\hline Fusarium semitectum & 5 & 4 & 5 & 3 & 4 & 3 & 5 & 2 & 4 & 1 & 2 & 3 & 1 & 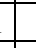 & 1 & - & - & - & - & 2 & 1 & - & - & - & - \\
\hline Fusarium solani & - & - & 2 & 1 & 1 & 1 & - & - & - & - & 3 & 4 & 5 & 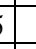 & 3 & - & - & - & - & - & - & 1 & 1 & - & - \\
\hline Fusarium moniliforme & - & - & 2 & 3 & 1 & 1 & 3 & 2 & 4 & 1 & 1 & 2 & - & & - & 1 & 1 & - & - & - & - & - & - & - & - \\
\hline Myrothecium roridum & - & - & - & - & 1 & 1 & - & - & 1 & 2 & - & - & - & & - & - & - & - & - & - & - & - & - & - & - \\
\hline Dark Sterile mycelium & 5 & 4 & 3 & 2 & 5 & 3 & - & - & 5 & 3 & 4 & 2 & 3 & 3 & 4 & 3 & 5 & - & - & 2 & 1 & 1 & 1 & - & - \\
\hline White Sterile mycelium & - & - & 2 & 1 & - & - & 3 & 2 & - & - & - & - & 2 & & 2 & - & - & 1 & 1 & - & - & - & - & - & - \\
\hline Unidentified I & - & - & - & - & - & - & - & - & - & - & - & - & 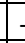 & & - & 1 & 1 & - & - & 1 & 2 & - & - & - & - \\
\hline Unidentified 2 & - & - & - & - & - & - & 1 & 1 & - & - & 1 & 2 & & & - & - & - & - & - & - & - & - & - & - & \\
\hline
\end{tabular}

Groups I: Dominant Fungi: This group includes only those decomposing mycoflora which appeared in more than six observations. Rhizopus stolonifer, Phoma glomerata, Aspergillus flavus, A. fumigatus, A. niger, A. sulphureus, Penicillium citrinum, P. rubrum, Trichoderma harzianum, Gliocladium roseum, Alternaria alternata, Curvularia lunata, Cladosporium cladosporioides, Fusarium semitectum and Dark sterile mycelium were found to be dominant fungi during isolation period.

Group II: Common Fungi: This group includes the fungi which occurred in five to six months during experimental period with less frequency and abundance. The common fungi isolated were Aspergillus terreus, Trichoderma koningii, Drechslera avanacea, Torula graminis, Fusarium solani and F. moniliforme.

Group III: Frequent Fungi: This group includes the mycoflora which appeared in at least 3-4 observations with normal frequency and abundance. Mucor racemosus, Chaetomium globosum, Macrophomina phaseoli, Pestolotia mangiferae, Aspergillus sydowi, Alternaria solani, Epicoccum purpourascens and white sterile mycelium were recorded as frequent fungi.
Group IV: Rare Fungi: The fungi of this group appeared only once or twice with very low frequency and abundance. Pythium aphanidermatum, Aspergillus luchuensis, Humicola grisea, Nigrospora sphaerica, Diplococcum spicatum and Myrothecium roridum were recorded to be the member of this group.

In present study, the fungi recorded from decomposing green manure substrate both in experimental and natural conditions were mostly the members of Dueteromycetes suggesting that the fungi belonging to this class are strong colonizers of the decaying substrate with better adaptability, high competitive ability, whereas those of Phycomycetes and Ascomycetes were found weak colonizers. These results are in accordance with De Santo et al. (2002) ${ }^{[6]}$ and Vibha and Sinha (2007) ${ }^{[35]}$. The dominance and predominance of Aspergillus spp., Penicillium citrinum, Fusarium spp, Trichoderma spp. were observed and obtained results were similar with the findings of Kara and Asan (2007) ${ }^{[13]}$, Pandey and Sinha (2008) ${ }^{[27]}$ and Kumar et al. (2011) ${ }^{[23]}$. The order of fungal succession upon a natural substrate reflects the sequential release of different organic and inorganic nutrients along with interaction between each individual and substratum besides the competition between individual fungi (Hobbie et al., 2003; Kodsueb et al., 2008; Sinha et al., 2010) ${ }^{[11,14,32]}$. 


\section{Conclusion}

The dominant decomposing fungi selected after screening based on several techniques, contribute efficiently in the decomposition process. Several fungal communities ensured their stage specific occurrence during the whole process of the decomposition. Their occurrence was fluctuated with the varying substrate of the decomposing green manure (Sesbania aculeata L.). Some of the dominant decomposing fungi viz., Aspergillus niger, Penicillium citrinum, Trichoderma harzianum, Curvularia lunata and Cladosporium cladosporioides contributed potentially in the decomposition of the Sesbania aculeata L. These fungi were already present in the soil but after getting their substrate in the form of Sesbania aculeata L. they enhanced their growth and reproduction by colonizing the substrate rapidly. These fungi can be used for the purpose of biodegradation of the agricultural waste and agro-industrial wastes.

\section{References}

1. Barnett HL, Hunter BB. Illustrated Genera of Imperfect Fungi, Burges Publishing Co., Minneapolis, Minnesota, USA, 1972.

2. Batty LC, Younger PL. The effect of $\mathrm{pH}$ on plant litter decomposition and metal cycling in wetland mesocosms supplied with mine drainage. Chemosphere. 2007; 66:158-164.

3. Berkenkamp A, Priesack E, Munch JC. Modeling the mineralization of plant residue on the soil surface, Agronomie. 2002; 22:711-722.

4. Bocock KL, Gilbert OJW. The disappearance of leaf litter under woodland condition, Plant and Soil. 1957; 9:179-185.

5. Boedijn KB. Trypon blue as a stain for fungi, Stain Technology. 1956; 31:115-116.

6. De Santo AV, Rutigliano FA, Berg B, Fioretto A, Puppi $\mathrm{G}$, Alfuni A et al. Fungal mycelium and decomposition of needle litter in three contrasting coniferous forests. Acta Oecologia. 2002; 23(4):247-259.

7. Ellis MB. More Dematiaceous Hyphomycetes, CMI, Kew, England, 1971.

8. Garrett SD. Soil Fungi and soil Fertility, The Macmillan Company, New York, 1981, 66-77.

9. Gilman JC. A Manual of soil Fungi, Oxford and IHB Publishing Co, Calcutta, 1975.

10. Herzog C, Hartmann M, Frey B, Stierli B, Rumpel C, Buchmann $\mathrm{N}$ et al. Microbial succession on decomposing root litter in a drought-prone Scots pine forest. ISME Journal. 2019; 13:2346-2362. doi: 10.1038/s41396-0190436-6

11. Hobbie EA, Watrud LS, Maggard S, Shiroyama T, Rygiewicz PT. Carbohydrate use and assimilation by litter and soil fungi assessed by carbon isotopes and BIOLOG (R) assays. Soil Biology and Biochemistry. 2003; 35(2):303-311.

12. Jing T, Shibata A, Zhou Q, Katayama A. Effect of temperature on reaction rate and microbial community in composting of cattle manure with rice straw, Journal of Bioscience and Bioengineering. 2007; 104(4):321-328.

13. Kara O, Asan A. Microfungal community structure from forest soils in Northern Thrace Region, Turkey. Annals of Microbiology. 2007; 57(2):149-155.

14. Kodsueb R, McKenzie EHC, Lumyong S, Hyde KD. Fungal succession on woody litter of Magnolia liliifera (Magnoliaceae). Fungal Diversity. 2008; 30:55-72.
15. Krishna MP, Mohan M. Litter decomposition in forest ecosystems: a review. Energy, Ecology and Environment. 2017; 2:236-249. doi: 10.1007/s40974-017-0064-9.

16. Kumar R, Sinha A, Srivastava S, Srivastava M. Effect of green manuring of Sesbania aculeata L. on rhizosphere microflora of okra (Abelmoschus esculentus L.). Crop Research. 2013; 46(1, 2-3):200-204.

17. Kumar S Samiksha, Sukul P. Green Manuring and Its Role in Soil Health Management. In: Giri B., Varma A. (eds) Soil Health. Soil Biology, vol 59. Springer, Cham, 2020. https://doi.org/10.1007/978-3-030-44364-1_13

18. Kumar R. Studies on decomposing mycoflora of Sesbania aculeata L. in soil and its effects on soil-borne plant pathogens. Ph. D. thesis, Banaras Hindu University, Varanasi, Uttar Pradesh, India, 2010.

19. Kumar R, Sinha A, Srivastava S, Mahajan G. Evaluation of biocontrol potential of some fungal decomposers of Sesbania aculeata L. green manure against some soilborne plant pathogens. Journal of Environmental Biology. 2017; 38:37-45.

20. Kumar R, Mahajan G, Srivastava S, Sinha A. Green Manuring: A boon for sustainable agriculture and pest management - A Review. Agri. Reviews. 2014a; 35(3):196-206.

21. Kumar R, Sinha A, Srivastava M, Srivastava S, Chattopadhyay A. Effect of Temperature on Production of Endoglucanase and Xylanase from Some Green Manure Fungal Decomposers. Annual Research \& Review in Biology. 2018; 25(5):1-7.

22. Kumar R, Sinha A, Srivastava S, Singh S. Evaluation of substrates for mass multiplication of green manure associated fungi for biological control of soil borne phytopathogens. Indian Phytopath, 2014b; 67(4):396401.

23. Kumar R, Sinha A, Srivastava S, Srivastava M. Variation in Soil Mycobiota Associated with Decomposition of Sesbania aculeata L. Asian Journal of Plant Pathology. 2011; 5:37-45.

24. Kumar R, Srivastava S, Srivastava M, Sinha M. Effect of organic amendments on soil mycoflora. Asian Journal of Plant Pathology. 2010; 4(2):73-81.

25. Manzoni S, Schimel JP, Porporato A. Responses of soil microbial communities to water stress: results from a meta-analysis. Ecology. 2012; 93(4):930-938.

26. Oladoye AO, Adams BA, Adedire MO, Agboola DA. Nutrient Dynamics and Litter Decomposition in Leucaena leucocephala (Lam.) De Wit Plantation in the Nigerian Derived Savanna. West African Journal of Applied Ecology. 2007; 13:1-17.

27. Pandey V, Sinha A. Mycoflora associated with decomposition of rice stubble mixed with soil. Journal of Plant Protection Research. 2008; 48(2):247-253.

28. Raper KB, Thom C. A Manual of Penicillia. Williams and Wilkins Co., Baltimore, USA, 1949.

29. Saksena SB. Ecological factors governing the distribution of soil microfungi in some forest soils of Sagar, Journal of Indian Botanical Society. 1955; 34:262-298.

30. Singh NS, Sharma R, Parween T, Patanjali PK. Pesticide contamination and human health risk factor. In: M. Oves et al. (eds.). Modern age environmental problems and their remediation, 2018. doi: 10.1007/978-3-319-645018 3.

31. Sinha A, Kumar R, Kamil D, Kapur P. Release of nitrogen, phosphorus and potassium from decomposing Crotalaria juncea L. in relation to different climatic 
factors, Environment and Ecology. 2009; 27(4B):20772081.

32. Sinha A, Srivastava M, Kumar R, Srivastava S, Mishra HM. Mycoflora of decomposing kitchen waste in relation to different climatic factors and its effect on soil borne plant pathogens. Environment and Ecology. 2010; 28(3):1458-1462.

33. Subramanium CV. Hyphomycetes, ICAR, New Delhi, 1971.

34. Thom C, Raper KB. Manual of Aspergilli. Williams and Wilkins Co., Baltimore, USA, 1945.

35. Vibha, Sinha A. Variation of soil mycoflora of rice stubble from rice wheat cropping system. Mycobiology. 2007; 35(4):191-195.

36. Warcup JH. Method for isolation and estimation of activities of fungi in soil, In: Parkinson D., Waids J.S., (Eds) Ecology of Soil Fungi, The University Press, Liverpool, 1960, 3-21.

37. Wardle DA, Bardgett RD, Walker LR, Bonner KI. Among and within species variation in plant litter decomposition in contrasting long-term chronosequences. Functional Ecology. 2009; 23(2): 442-453.

38. Xu RK, Coventry DR. Soil pH changes associated lupin and Wheat plant materials incorporated in a red-brown soil. Plant and Soil. 2003; 250(1):113-119.

39. Zaller JG, Koepke U. Effect of traditional and bio dynamic farmyard manure amendment on yields, soil chemical, biochemical and biological properties in a long term field experiment. Biology Fertility of Soils. 2004; 40:222-229. 